\title{
Microlensing: Exploring the Dark Corners of the Galaxy with a Thousand Tiny Flashlights
}

\author{
Jennifer C. Yee* \\ $\dagger$ \\ Harvard-Smithsonian Center for Astrophysics \\ E-mail: jyeedcfa.harvard.edu
}

In microlensing, the light from a background source star is bent by the gravitational potential of an intervening lens. The resulting light curve reveals the nature of the lens. Because no light from the lens itself is necessary to make this measurement, microlensing is able to probe both faint and dark objects, including distant planetary systems and black holes. The advent of high-cadence, wide-field microlensing surveys and the first microlensing programs in space have revolutionized the field and opened new areas of investigation including the search for dark binaries and understanding planet occurrence in different Galactic environments. I will review recent results from these programs and their potential to help us understand Galactic structure.

Frank N. Bash Symposium 2015

18-20 October

The University of Texas at Austin, USA

\footnotetext{
* Speaker.

†Sagan Fellow; Work by J.C.Y. was performed under contract with the California Institute of Technology (Caltech) / Jet Propulsion Laboratory (JPL) funded by NASA through the Sagan Fellowship Program executed by the NASA Exoplanet Science Institute.
} 


\section{Introduction}

\subsection{Essentials of Microlensing}

Microlensing is the gravitational lensing of one star's light by another star. At least this is how it was originally conceived by Einstein in his 1936 paper "Lens-like Action of a Star by the Deviation of Light in a Gravitational Field" [1]]. This is a good starting point for understanding microlensing, but as we will see, microlensing encompasses far more than just stars ${ }^{1}$.

Einstein (1936) provides a simple description of microlensing. First, if two stars at arbitrary distances are perfectly aligned along the line of sight, the light from the background star will appear as a ring with radius

$$
\theta_{\mathrm{E}}=\sqrt{\kappa M_{\mathrm{L}} \pi_{\text {rel }}} \quad \text { where } \quad \pi_{\text {rel }}=\frac{\mathrm{AU}}{D_{\mathrm{L}}}-\frac{\mathrm{AU}}{D_{\mathrm{S}}} \quad \text { and } \quad \kappa=8.14 \operatorname{mas}_{\odot}^{-1} .
$$

The size of $\theta_{\mathrm{E}}$ depends on both the lens mass $M_{\mathrm{L}}$ and the distances to the lens and source, $D_{\mathrm{L}}$ and $D_{\mathrm{S}}$, respectively. Second, if the alignment is less than perfect, i.e. the stars appear ever-so-slightly displaced from each other, instead of a perfect ring the light will be split into two images, major and minor (aka large and small) located at

$$
y_{ \pm}= \pm \frac{1}{2}\left(u \pm \sqrt{u^{2}+4}\right)
$$

where $u$ is the separation between the source and lens as a fraction of the Einstein radius. Figure 1 illustrates these two images. Although the radius of the Einstein ring, and there for the separation of these two images, is too small ( $\theta_{\mathrm{E}} \sim 1$ mas) to be resolved even with modern telescopes, there is still an overall magnification effect

$$
A(u)=\frac{u^{2}+2}{u \sqrt{u^{2}+4}} .
$$

Later papers by Tikhov (1938), Liebes (1964), and Refsdal (1964) expand the theory of microlensing [1, 4,5$]^{2}$. In particular, they discuss how microlensing would be observed in practice. Our galaxy contains billions of stars all moving on their own individual orbits. As the Sun executes its own orbit through the Galaxy, we see the other stars moving relative to each other like cars on a highway. Sometimes those stars come into conjunction with each other, i.e. two stars at very different distances will align with each other along our line of sight. As this happens, a microlensing event occurs. The lens-source separation in Equations 1.2 and 1.3 is a function of time. As shown in Figure 1 the images therefore change as a function of $u$ and hence as a function of time, leading to a varying magnification. The microlensing light curve is the observed change in brightness due to the lensing effect as a function of time.

\subsection{Microlensing as a Type of Gravitational Lensing}

Because microlensing results in multiple images of the background source, it is a subset of strong lensing. However, microlensing differs from galaxy-cluster scale lensing in one important

\footnotetext{
${ }^{1}$ Also note that there is a separate branch of microlensing that studies the effects of individual stars of a galaxy lens on the images of a multiply-imaged quasar (e.g. see Section 7 of [目]).

${ }^{2}$ The reader is also referred to more comprehensive reviews of modern microlensing (e.g. 过, 6, 团).
} 


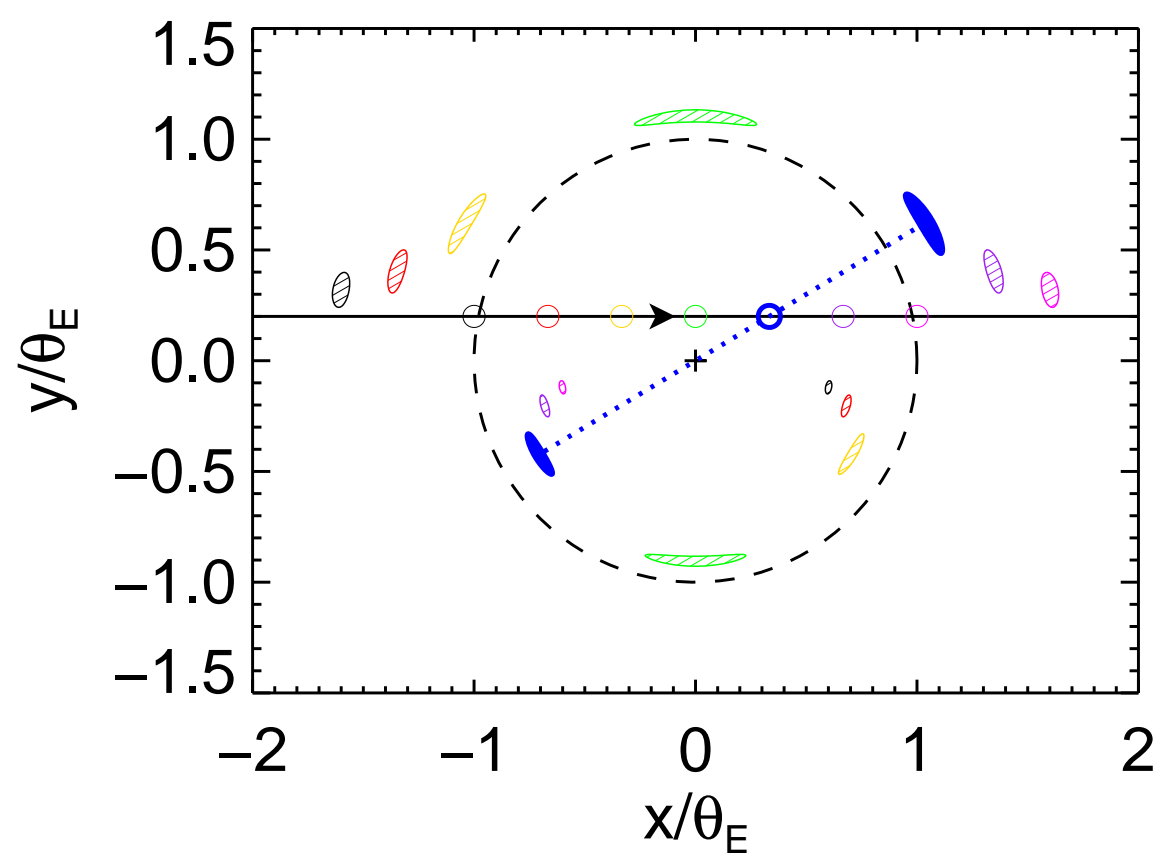

Figure 1: A face-on view of microlensing. The background source star (circle) is shown traveling from left to right relative to the lens star (+). The corresponding images of the source are also shown. The case shown in blue emphasizes that the images of the source appear along the line between the source and lens with the larger, major image just outside the Einstein ring (dashed black circle) and the smaller, minor image just inside the Einstein ring.

way. Usually, when we think of strong lensing, we think of how the gravitational lensing effect which enables us to study distant galaxies and quasars at large $z$ by amplifying their light. This is the study of the background source, i.e. the object being imaged. In contrast, for microlensing, the interest is in the lens. The form of the lightcurve (and the underlying images) reflects the gravitational potential of the lens star. The goal is to reconstruct information about the lens by modeling the shape of the lightcurve. The nature of the source and details of its light are irrelevant ${ }^{3}$. In this sense, the source is analogous to a flashlight: it is just a tool used to study the lens.

\section{Applications of Microlensing}

So far we have assumed that the lens is a star, but the underlying theory depends only on the assumption that the lens is a point mass (or a collection of point masses). In fact, the very first microlensing searches were carried out not to detect microlensing with stars, but to determine whether or not Massive Compact Halo Objects could account for dark matter [11, 12, 13]. This application reflects the power of microlensing: it can detect objects regardless of their light output, including objects that do not emit light. Hence, microlensing can be used to find stellar mass black

\footnotetext{
${ }^{3}$ The exception is work by Bensby et al. that took advantage of the magnification effect to take spectra of dwarf stars in the Bulge [8, 9, 10]
} 
holes and other stellar remnants that are isolated or in wide binaries. It can also find brown dwarfs or free-floating planets.

The primary motivation of modern microlensing surveys is the search for planets orbiting the lens stars, so I will begin by discussing planetary microlensing in detail. However, on a broader scale microlensing offers a unique way to study galactic structure. Consider that microlensing is a random process depending on the motions of individual objects within the Galaxy. Then the underlying population of microlenses reflects the underlying population of the Galaxy. A complete statistical analysis of a microlensing survey has the potential to tell us about the complete mass function of the Galaxy ranging from free-floating planets all the way up to isolated black holes.

\subsection{Planetary Microlensing}

To first order, planetary companions to the lens star can be thought of as perturbations. The lens star is far more massive, so it will dominate the deflection of the light and hence, the microlensing light curve. However, if there is a planet near one of the images, it will further deflect the light, distorting the image and the light curve [14, 15]. Hence, the sensitivity of an individual event to planets, depends on the paths traced by the images, which in turn reflects the path of the source. Additionally, if the lens star is perfectly (or nearly perfectly) aligned with the source, such that the source is imaged into a ring, that event will be extremely sensitive to planets because such large images are easily perturbed and have complete angular coverage about the lens star [16].

An alternative way to think about microlensing is through the magnification map. We have a mathematical description for the magnification produced by a particular lens. Using Equation 1.3, we can calculate what the magnification of a source will be at any point relative to a lens star. For a point lens, this map has the basic properties of being radially symmetric, being larger with smaller $u$, and diverging to infinity as $u \rightarrow 0$. A planet will perturb this map as in Figure 2. To a first approximation, the strongest perturbations happen at source locations that place one of the images at the location of the planet. Along the "caustics," the magnification will diverge to infinity. Note that there are usually two sets of caustics, the planetary and central caustics. A planetary caustic reflects a perturbation of an image created when the source is well-separated from the lens, whereas the central caustic reflects the perturbation of an exceptionally large, ring-like image created when the source is directly behind the lens star.

Any number of microlensing light curves can be created given a magnifcation map for a particular lens by tracing different paths through that map. For example the path shown in Figure 2 maps onto the light curve shown in Figure 3 . The main difficulty in microlensing is reconstructing the underlying map (and lens system) based on only the light curve, which is effectively a 1D slice of a 2D map. This leads to a number of degeneracies, which may or may not be resolved by a full model. These degeneracies range from the trivial to the severe (see for example [18, 19, 20, 21]). For example, the magnification map is symmetric across the star-planet axis, meaning that two different source trajectories can give rise to the observed light curve. Whether or not this matters to the interpretation of the lens depends on the circumstances. Alternatively, sometimes the light curve can be explained by more than one lens system. 


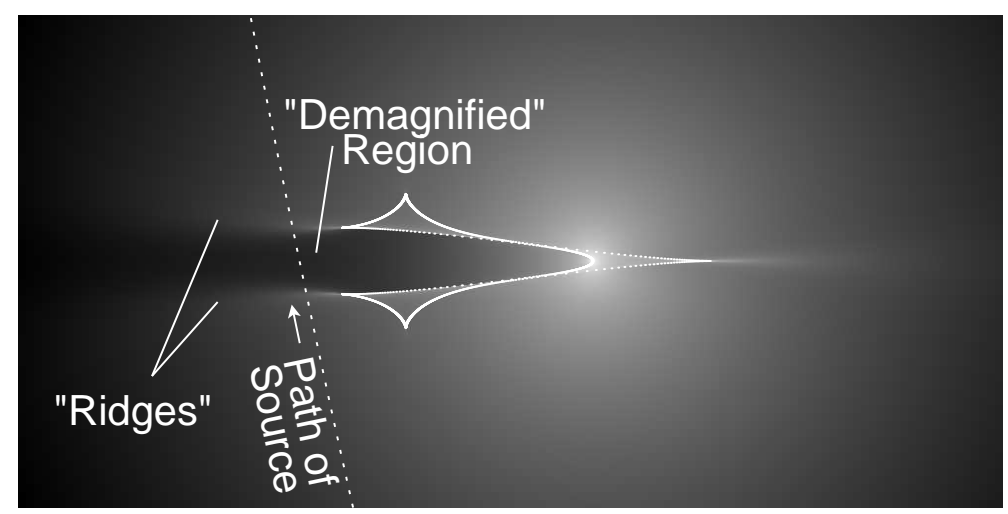

Figure 2: Magnification map for OGLE-2014-BLG-0124 [17]. The presence of the planet distorts the underlying magnification pattern due to the lens star. The caustic is the white outline; in this case the central and planetary caustics are merged into a single, resonant caustic. The path of the source produces the light curve shown in Figure 3.

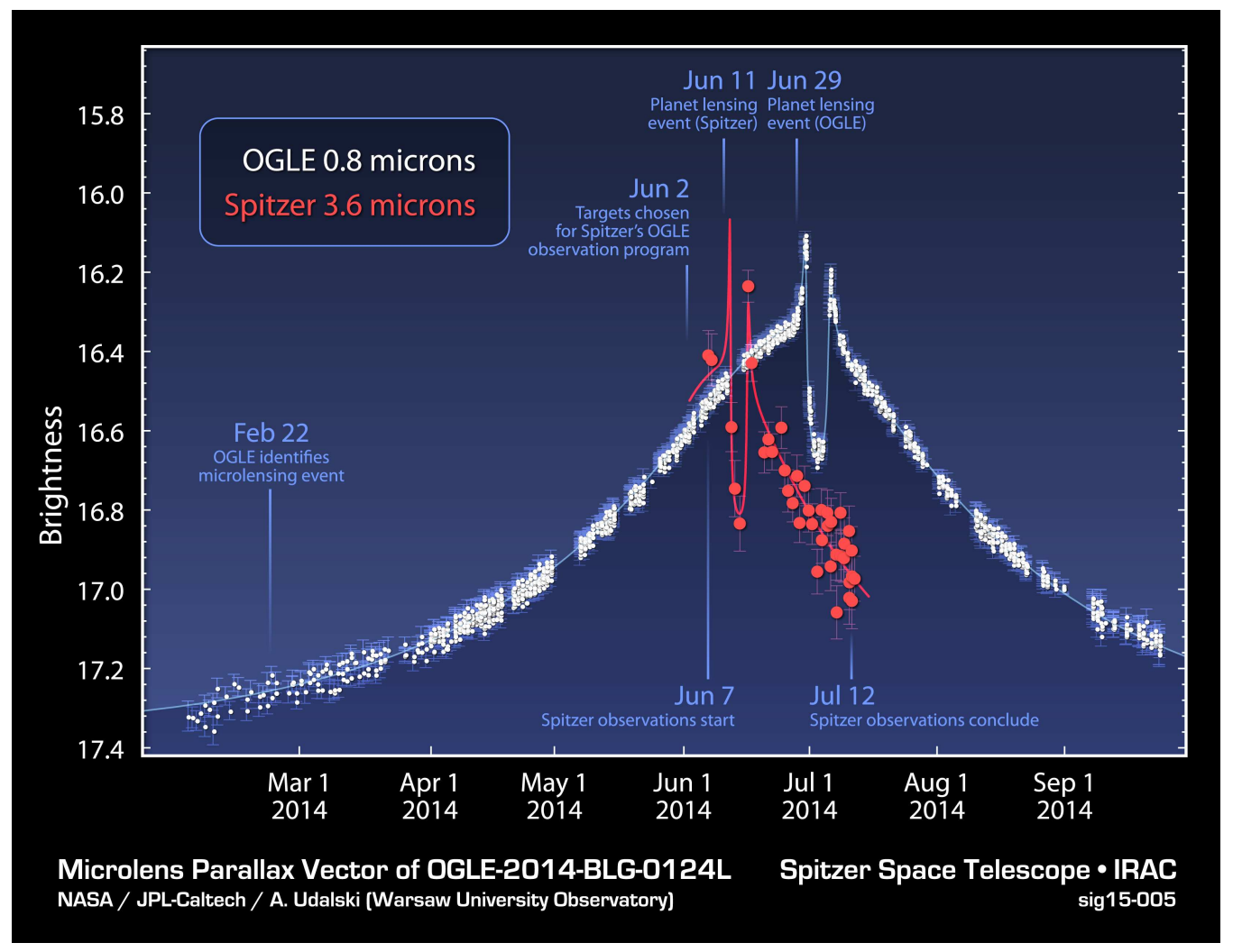

Figure 3: The light curves of OGLE-2014-BLG-0124 as seen from OGLE (white) and Spitzer (red). The planetary perturbation is seen from Spitzer $\sim 20$ days before it is seen from Earth because of the microlens parallax effect. Image credit: NASA/JPL-Caltech/Warsaw University Observatory. 


\section{Modern Microlensing Surveys}

Observational microlensing is shaped by the tension between the rarity of microlensing events and the fleeting nature of planetary perturbations. Even in the densest stellar fields, i.e. in the direction of the Galactic Bulge, the probability of a microlensing event is one in a million [22]. At the same time, the typical planetary perturbations last between a few hours and a few days [14, 15]. This means that conducting an ideal microlensing survey requires monitoring millions of stars several times per hour, 24 hours per day. In the past, this was impossible, so the work was split between surveys with cadences of $\sim 1 \mathrm{day}^{-1}$ to detect events and followup teams, which would monitor some subset of microlensing events at a much higher cadence. This strategy has resulted in the majority microlensing planet discoveries to date. However, it makes statistical analyses difficult because of the complex selection function. While some statistical analyses have been done (e.g. [23, 24, 25, 26, 27]), a truly objective microlensing survey would yield more precise and more complete results.

Over the past decade, there has been a major push to increase the size of the cameras used for microlensing surveys to increase the field of view and therefore the cadence at which microlensing events can be observed. The goal is to image a large enough area of the sky to detect large numbers of microlensing events while observing them at a high enough cadence to detect and characterize planetary perturbations. In the past couple of years this goal has been achieved, and surveys will soon eclipse followup groups as the primary sources of planet detections.

There are four modern microlensing surveys: OGLE, MOA, Wise, and KMTNet. The Optical Gravitational Lensing Experiment (OGLE) has a 1.3m telescope based at Las Campanas Observatory and it is the most powerful, single site microlensing survey currently operating [28]. In the 2015 microlensing season, OGLE IV discovered 2145 microlensing events ${ }^{4}$, which may be contrasted with the dozens of events discovered annually by OGLE II in the late 1990s or the hundreds of events per year from OGLE III in the 2000s . The Microlensing Observations in Astrophysics (MOA) survey has a telescope based at Mt. John Observatory in New Zealand [29]. In 2015, they found 576 microlensing events with some overlap with the OGLE survey. Wise Observatory in Israel ${ }^{5}$ is also conducting a microlensing survey, but they do not issue real-time microlensing alerts [30, 27]. Finally, the Korea Microlensing Telescope Network was commissioned in 2015 [31]. It has $1.6 \mathrm{~m}$ telescopes located at three sites: CTIO in Chile, SAAO in South Africa, and SSO in Australia. This global distribution of telescopes combined with the large field of view (4 square degrees) means that this survey can monitor 16 square degrees continuously with a 10 minute cadence, which is more than sufficient to detect Earth-mass ratio exoplanets [31, 32]. These new and upgraded surveys have and are continuing to revolutionize the field of microlensing.

\section{Recent Microlensing Discoveries}

\subsection{Examples of Planetary Discoveries}

The larger fields-of-view mean more data and more data means that microlensing events are being discovered earlier in their evolution and earlier in the microlensing season. In the second

\footnotetext{
${ }^{4}$ http://ogle.astrouw.edu.pl/ogle4/ews/2015/ews.html

${ }^{5}$ Note that this is unrelated to the Wide-field Infrared Survey Explorer (WISE).
} 
full season of OGLE-IV, the OGLE Early Warning System announced the discovery of OGLE2012-BLG-0026 on February 13, when the Bulge is visible for $\lesssim 2 \mathrm{hr} \mathrm{night}^{-1}$. Because of this early alert, we were able to track the evolution of this event and recognize that it would be a highmagnification microlensing event. By mobilizing telescope resources around the globe, especially the Microlensing Follow-Up Network (MicroFUN), we obtained dense coverage of the event over peak. As a result, two planets with mass ratios $q=1.3 \times 10^{-4}$ and $q=8 \times 10^{-4}$ were discovered [33]. This is an example of how followup groups and surveys work together.

However, the case of OGLE-2012-BLG-0406 illustrates how followup data is becoming less important. Poleski et al. (2014) show that this $q \sim 6 \times 10^{-3}$ planetary perturbation was adequately covered by the OGLE-IV survey data alone [34]. The addition of dense followup data decreases the uncertainties [35], but the OGLE data alone capture the basic properties of the event.

A faster cadence also means routine characterization of shorter timescale microlensing phenomena. This includes the possibility of detecting free-floating planets, which produce events lasting only a few hours. Sumi et al. (2011) studied the distribution of event timescales from MOA-II [36]. They found an excess of short timescale microlensing events that cannot be accounted for by the stellar population. They posited an additional population of free-floating planets to explain this excess. This comprehensive analysis of microlensing survey data is an early example of what we can expect from surveys in the future.

\subsection{Microlensing Parallax}

The biggest uncertainty in microlensing is that the shape of the light curve alone only gives relative information about the lens. For example, modeling a planetary perturbation will yield the mass ratio between the planet and the host star, but it will not directly yield the mass of that host star. As long as the mass ratio is small enough $\left(q \lesssim 10^{-3}\right)$, the system obviously contains a planet, but the exact nature of the planet is unknown. However, there is also a significant gray area (e.g. $q \sim 10^{-2}$ ) for which the planetary nature of the object is ambiguous without knowing the mass of the host.

One way to overcome this challenge is to measure higher-order effects in the microlensing light curve. Measuring both the angular size of the Einstein ring through finite source effects and microlens parallax $\pi_{\mathrm{E}}$ allows a direct measurement of the lens mass and its distance:

$$
M_{\mathrm{L}}=\frac{\theta_{\mathrm{E}}}{\kappa \pi_{\mathrm{E}}} \quad ; \quad D_{\mathrm{L}}^{-1}=\theta_{\mathrm{E}} \pi_{\mathrm{E}}+D_{\mathrm{S}}^{-1}
$$

$D_{\mathrm{S}}$ is the distance to the source, which can generally be assumed to be $8 \mathrm{kpc}$.

Finite source effects are literally the effect on the light curve attributable to the fact that the source is a star with a finite size rather than a perfect point source. The observed magnification of the source's light is the integration of the magnification pattern across the face of the source star. Hence, if the source crosses a caustic, instead of observing the magnification diverge to infinity, this feature is rounded out. The degree of rounding gives a measurement of the source size $\rho=\theta_{\star} / \theta_{\mathrm{E}}$. Since the angular size of the source $\theta_{\star}$ can be inferred from the source color and surface brightness relations [37], this yields a measurement of $\theta_{\mathrm{E}}$.

In microlensing, the microlens parallax vector gives the displacement of the lens-source trajectory from the expectation of rectilinear motion observed from a single location. This is related 
to the physical parallax by

$$
\pi_{\mathrm{E}}=\frac{\pi_{\mathrm{rel}}}{\theta_{\mathrm{E}}}
$$

There are two ways of observing parallax effects in microlensing. First, observations are generally not taken from an inertial frame. The Earth and various space telescopes are all accelerating platforms. This acceleration can be seen in the light curve of the microlensing event, provided the event timescale is a significant fraction of the orbit [38, 39, 40]. Second, a microlensing event from two different locations will have a different observed light curve for each location because of the parallax effect [41, 42, 43, 44,

One major result from measuring lens masses through parallax and finite source effects was the discovery of brown dwarf binaries. Microlensing has found some of the lowest-mass brown-dwarfbrown-dwarf binaries. One example is OGLE-2009-BLG-151/MOA-2009-BLG-232 in which the primary is $0.018 M_{\odot}$ and the secondary is $0.0075 M_{\odot}$ [45]. Figure 5 of [46] shows these binaries in context with other known low-mass binaries.

\subsection{Spitzer Parallaxes}

Recently, Spitzer time has been allocated to microlens parallax experiments, vastly increasing the number of events with parallax measurements. The Einstein ring projected onto the observer plane is typically about 10 AU. Because Spitzer is $\sim 1$ AU from the Earth (a significant fraction of the Einstein ring size), it observes a different apparent alignment between the source and lens stars, and hence, a different light curve.

So far, the 2014 and 2015 Spitzer Microlensing Campaigns have observed over 200 microlensing events [47, 48]. In 2014, we demonstrated the feasibility of measuring microlens parallaxes with Spitzer by observing $\sim 60$ microlensing events. In this campaign, we made the first mass measurement for an isolated star using space-based microlens parallax observations [49]. We also made the first space-based parallax measurement for a microlensing planet [17]. Figure 3 shows the light curves of this event as observed from the ground and from Spitzer, as well as the sequence of events for scheduling observations for this event. The $\sim 20$ day offset between the two light curves is due to the parallax effect. Note that all decisions were made without reference to the Spitzer light curve since those data were not reduced until after the campaign ended, so the fact that the planetary perturbation was captured by the Spitzer data was entirely fortuitous. The 2015 campaign has already yielded a microlens parallax measurement for a second planet (OGLE2015-BLG-0966 [50]). In addition, we discovered a wide binary whose primary is likely to be a non-accreting stellar remnant. The parallax measurement from Spitzer was crucial for constraining the mass of the primary, which identifies it as a likely stellar remnant [51].

In addition to these individual discoveries, the Spitzer campaign has opened new possibilities for statistical studies from microlensing. The microlens parallax measurement itself yields a very good statistical estimate of the distance to the individual lenses, and when combined with finite source effects (which are usually measured for planets and binaries) yields a precise measurement of the lens distance (Equation 4.1). With these distance measurements, we can locate the individual lenses in the Galaxy, effectively mapping out the underlying population of objects including those that do not emit light (e.g. black holes). One of the major goals of these campaigns is to make the first measurement of the distribution of planets as a function of Galactic distance, and test the 
effect of galactic environment on planet formation by comparing the relative abundance of planets in the Disk and the Bulge [47, 52].

In 2016, there will be a third Spitzer microlens parallax campaign [53, 54]. In addition, Campaign 9 of the $K 2$ mission (K2C9), also in 2016, will use the Kepler satellite to conduct a microlens parallax campaign [40, 55]. While the Spitzer campaigns are targeted to specific microlensing events discovered from the ground, the $K 2$ campaign will take advantage of the large field-of-view to conduct a survey-like experiment. $K 2$ will download a large superstamp for the duration of the 80-day campaign. The primary advantage of this approach is that $K 2 C 9$ will be able to measure parallaxes for shorter timescale microlensing events that occur in the superstamp. This is a particularly interesting opportunity for measuring parallaxes of candidate free-floating planet events (timescales of $\sim 1$ day). Such events cannot be observed by the Spitzer program because the lag time between scheduling and the start of observations is too long.

\section{The Future of Microlensing}

Looking farther into the future, the WFIRST satellite is expected to launch in 2024 [56]. Thirty percent of this mission will be dedicated to a microlensing survey of the Galactic Bulge. The WFIRST microlensing mission will discover planets on a scale similar to the Kepler mission. The highly precise photometry available from space will lead to the discovery of thousands of exoplanets. This mission will complement other search techniques by finding planets as small as Mercury at distances of a few AU; planets this small and distant cannot be found by any other technique. Furthermore, many of these planets will have precise masses and known Galactic distances. [57] shows how WFIRST will combine various pieces of information, including microlens parallax, to obtain precise mass and distance measurements for its lenses. This mission will revolutionize the field of microlensing and our understanding of planets beyond the snow line.

\section{References}

[1] A. Einstein, Lens-Like Action of a Star by the Deviation of Light in the Gravitational Field, Science 84 (Dec., 1936) 506-507.

[2] J. Wambsganss, Part 4: Gravitational microlensing, in Saas-Fee Advanced Course 33: Gravitational Lensing: Strong, Weak and Micro (G. Meylan, P. Jetzer, P. North, P. Schneider, C. S. Kochanek, and J. Wambsganss, eds.), pp. 453-540, 2006.

[3] G. A. Tikhov, Sur la déviation des rayons lumineux dans le champ de gravitation des étoiles, Mitteilungen der Nikolai-Hauptsternwarte zu Pulkowo 16 (1938) A1-A22.

[4] S. Liebes, Gravitational Lenses, Physical Review 133 (Feb., 1964) 835-844.

[5] S. Refsdal, The gravitational lens effect, MNRAS 128 (1964) 295.

[6] B. S. Gaudi, Exoplanetary Microlensing, ArXiv e-prints (Feb., 2010) [arXiv:1002.0332].

[7] B. S. Gaudi, Microlensing Surveys for Exoplanets, ARA\&A 50 (Sept., 2012) 411-453.

[8] T. Bensby, S. Feltzing, J. A. Johnson, A. Gould, D. Adén, M. Asplund, J. Meléndez, A. Gal-Yam, S. Lucatello, H. Sana, T. Sumi, N. Miyake, D. Suzuki, C. Han, I. Bond, and A. Udalski, Chemical evolution of the Galactic bulge as traced by microlensed dwarf and subgiant stars. II. Ages, 
metallicities, detailed elemental abundances, and connections to the Galactic thick disc, A\&A $\mathbf{5 1 2}$ (Mar., 2010) A41+, arXiv:0911.5076.

[9] T. Bensby, D. Adén, J. Meléndez, A. Gould, S. Feltzing, M. Asplund, J. A. Johnson, S. Lucatello, J. C. Yee, I. Ramírez, J. G. Cohen, I. Thompson, I. A. Bond, A. Gal-Yam, C. Han, T. Sumi, D. Suzuki, K. Wada, N. Miyake, K. Furusawa, K. Ohmori, T. Saito, P. Tristram, and D. Bennett, Chemical evolution of the Galactic bulge as traced by microlensed dwarf and subgiant stars. IV. Two bulge populations, A\&A 533 (Sept., 2011) A134+, arXiv:1107.5606.

[10] T. Bensby, J. C. Yee, S. Feltzing, J. A. Johnson, A. Gould, J. G. Cohen, M. Asplund, J. Meléndez, S. Lucatello, C. Han, I. Thompson, A. Gal-Yam, A. Udalski, D. P. Bennett, I. A. Bond, W. Kohei, T. Sumi, D. Suzuki, K. Suzuki, S. Takino, P. Tristram, N. Yamai, and A. Yonehara, Chemical evolution of the Galactic bulge as traced by microlensed dwarf and subgiant stars. V. Evidence for a wide age distribution and a complex MDF, A\&A 549 (Jan., 2013) A147, [arXiv:1211.6848].

[11] B. Paczynski, Gravitational microlensing by the galactic halo, ApJ 304 (May, 1986) 1-5.

[12] C. Alcock, R. A. Allsman, D. R. Alves, T. S. Axelrod, A. C. Becker, D. P. Bennett, K. H. Cook, N. Dalal, A. J. Drake, K. C. Freeman, M. Geha, K. Griest, M. J. Lehner, S. L. Marshall, D. Minniti, C. A. Nelson, B. A. Peterson, P. Popowski, M. R. Pratt, P. J. Quinn, C. W. Stubbs, W. Sutherland, A. B. Tomaney, T. Vandehei, and D. Welch, The MACHO Project: Microlensing Results from 5.7 Years of Large Magellanic Cloud Observations, ApJ 542 (Oct., 2000) 281-307, [astro-ph/0001272].

[13] T. Lasserre, C. Afonso, J. N. Albert, J. Andersen, R. Ansari, É. Aubourg, P. Bareyre, F. Bauer, J. P. Beaulieu, G. Blanc, A. Bouquet, S. Char, X. Charlot, F. Couchot, C. Coutures, F. Derue, R. Ferlet, J. F. Glicenstein, B. Goldman, A. Gould, D. Graff, M. Gros, J. Haissinski, J. C. Hamilton, D. Hardin, J. de Kat, A. Kim, É. Lesquoy, C. Loup, C. Magneville, B. Mansoux, J. B. Marquette, É. Maurice, A. Milsztajn, M. Moniez, N. Palanque-Delabrouille, O. Perdereau, L. Prévot, N. Regnault, J. Rich, M. Spiro, A. Vidal-Madjar, L. Vigroux, S. Zylberajch, and EROS Collaboration, Not enough stellar mass Machos in the Galactic halo, A\&A 355 (Mar., 2000) L39-L42, [astro-ph/0 002253 ].

[14] S. Mao and B. Paczynski, Gravitational microlensing by double stars and planetary systems, ApJL 374 (June, 1991) L37-L40.

[15] A. Gould and A. Loeb, Discovering planetary systems through gravitational microlenses, ApJ 396 (Sept., 1992) 104-114.

[16] K. Griest and N. Safizadeh, The Use of High-Magnification Microlensing Events in Discovering Extrasolar Planets, ApJ 500 (June, 1998) 37-+, astro-ph/.

[17] A. Udalski, J. C. Yee, A. Gould, S. Carey, W. Zhu, J. Skowron, S. Kozłowski, R. Poleski, P. Pietrukowicz, G. Pietrzyński, M. K. Szymański, P. Mróz, I. Soszyński, K. Ulaczyk, L. Wyrzykowski, C. Han, S. Calchi Novati, and R. W. Pogge, Spitzer as a Microlens Parallax Satellite: Mass Measurement for the OGLE-2014-BLG-0124L Planet and its Host Star, ApJ 799 (Feb., 2015) 237, arXiv:1410.4219].

[18] A. Gould, Resolution of the MACHO-LMC-5 Puzzle: The Jerk-Parallax Microlens Degeneracy, ApJ 606 (May, 2004) 319-325, [astro-ph/.

[19] D. P. Bennett, I. A. Bond, A. Udalski, T. Sumi, F. Abe, A. Fukui, K. Furusawa, J. B. Hearnshaw, S. Holderness, Y. Itow, K. Kamiya, A. V. Korpela, P. M. Kilmartin, W. Lin, C. H. Ling, K. Masuda, Y. Matsubara, N. Miyake, Y. Muraki, M. Nagaya, T. Okumura, K. Ohnishi, Y. C. Perrott, N. J. Rattenbury, T. Sako, T. Saito, S. Sato, L. Skuljan, D. J. Sullivan, W. L. Sweatman, P. J. Tristram, P. C. M. Yock, M. Kubiak, M. K. Szymański, G. Pietrzyński, I. Soszyński, O. Szewczyk, 
L. Wyrzykowski, K. Ulaczyk, V. Batista, J. P. Beaulieu, S. Brillant, A. Cassan, P. Fouqué, P. Kervella, D. Kubas, and J. B. Marquette, A Low-Mass Planet with a Possible Sub-Stellar-Mass Host in Microlensing Event MOA-2007-BLG-192, ApJ 684 (Sept., 2008) 663-683, arXiv: 0806.0025.

[20] J. C. Yee, Y. Shvartzvald, A. Gal-Yam, I. A. Bond, A. Udalski, S. Kozłowski, C. Han, A. Gould, J. Skowron, D. Suzuki, F. Abe, D. P. Bennett, C. S. Botzler, P. Chote, M. Freeman, A. Fukui, K. Furusawa, Y. Itow, S. Kobara, C. H. Ling, K. Masuda, Y. Matsubara, N. Miyake, Y. Muraki, K. Ohmori, K. Ohnishi, N. J. Rattenbury, T. Saito, D. J. Sullivan, T. Sumi, K. Suzuki, W. L. Sweatman, S. Takino, P. J. Tristram, K. Wada, the MOA Collaboration, M. K. Szymański, M. Kubiak, G. Pietrzyński, I. Soszyński, R. Poleski, K. Ulaczyk, L. Wyrzykowski, P. Pietrukowicz, the OGLE Collaboration, W. Allen, L. A. Almeida, V. Batista, M. Bos, G. Christie, D. L. DePoy, S. Dong, J. Drummond, I. Finkelman, B. S. Gaudi, E. Gorbikov, C. Henderson, D. Higgins, F. Jablonski, S. Kaspi, I. Manulis, D. Maoz, J. McCormick, D. McGregor, L. A. G. Monard, D. Moorhouse, J. A. Muñoz, T. Natusch, H. Ngan, E. Ofek, R. W. Pogge, R. Santallo, T.-G. Tan, G. Thornley, I.-G. Shin, J.-Y. Choi, S.-Y. Park, C.-U. Lee, J.-R. Koo, and the $\mu$ FUN Collaboration, MOA-2011-BLG-293Lb: A Test of Pure Survey Microlensing Planet Detections, ApJ 755 (Aug., 2012) 102, [arXiv:1201.1002].

[21] D. P. Bennett, V. Batista, I. A. Bond, C. S. Bennett, D. Suzuki, J.-P. Beaulieu, A. Udalski, J. Donatowicz, V. Bozza, F. Abe, C. S. Botzler, M. Freeman, D. Fukunaga, A. Fukui, Y. Itow, N. Koshimoto, C. H. Ling, K. Masuda, Y. Matsubara, Y. Muraki, S. Namba, K. Ohnishi, N. J. Rattenbury, T. Saito, D. J. Sullivan, T. Sumi, W. L. Sweatman, P. J. Tristram, N. Tsurumi, K. Wada, P. C. M. Yock, MOA Collaboration, M. D. Albrow, E. Bachelet, S. Brillant, J. A. R. Caldwell, A. Cassan, A. A. Cole, E. Corrales, C. Coutures, S. Dieters, D. Dominis Prester, P. Fouqué, J. Greenhill, K. Horne, J.-R. Koo, D. Kubas, J.-B. Marquette, R. Martin, J. W. Menzies, K. C. Sahu, J. Wambsganss, A. Williams, M. Zub, PLANET Collaboration, J. Y. Choi, D. L. DePoy, S. Dong, B. S. Gaudi, A. Gould, C. Han, C. B. Henderson, D. McGregor, C.-U. Lee, R. W. Pogge, I.-G. Shin, J. C. Yee, $\mu$ FUN Collaboration, M. K. Szymański, J. Skowron, R. Poleski, S. Kozłowski, L. Wyrzykowski, M. Kubiak, P. Pietrukowicz, G. Pietrzyński, I. Soszyński, K. Ulaczyk, OGLE Collaboration, Y. Tsapras, R. A. Street, M. Dominik, D. M. Bramich, P. Browne, M. Hundertmark, N. Kains, C. Snodgrass, I. A. Steele, The RoboNet Collaboration, I. Dekany, O. A. Gonzalez, D. Heyrovský, R. Kandori, E. Kerins, P. W. Lucas, D. Minniti, T. Nagayama, M. Rejkuba, A. C. Robin, and R. Saito, MOA-2011-BLG-262Lb: A Sub-Earth-Mass Moon Orbiting a Gas Giant Primary or a High Velocity Planetary System in the Galactic Bulge, ApJ 785 (Apr., 2014) 155, [arXiv:1312.3951].

[22] B. Paczynski, Gravitational microlensing of the Galactic bulge stars, ApJL 371 (Apr., 1991) L63-L67.

[23] B. S. Gaudi, M. D. Albrow, J. An, J.-P. Beaulieu, J. A. R. Caldwell, D. L. DePoy, M. Dominik, A. Gould, J. Greenhill, K. Hill, S. Kane, R. Martin, J. Menzies, R. M. Naber, J.-W. Pel, R. W. Pogge, K. R. Pollard, P. D. Sackett, K. C. Sahu, P. Vermaak, P. M. Vreeswijk, R. Watson, and A. Williams, Microlensing Constraints on the Frequency of Jupiter-Mass Companions: Analysis of 5 Years of PLANET Photometry, ApJ 566 (Feb., 2002) 463-499, astro-ph/.

[24] T. Sumi, D. P. Bennett, I. A. Bond, A. Udalski, V. Batista, M. Dominik, P. Fouqué, D. Kubas, A. Gould, B. Macintosh, K. Cook, S. Dong, L. Skuljan, A. Cassan, F. Abe, C. S. Botzler, A. Fukui, K. Furusawa, J. B. Hearnshaw, Y. Itow, K. Kamiya, P. M. Kilmartin, A. Korpela, W. Lin, C. H. Ling, K. Masuda, Y. Matsubara, N. Miyake, Y. Muraki, M. Nagaya, T. Nagayama, K. Ohnishi, T. Okumura, Y. C. Perrott, N. Rattenbury, T. Saito, T. Sako, D. J. Sullivan, W. L. Sweatman, P. J. Tristram, P. C. M. Yock, MOA Collaboration, J. P. Beaulieu, A. Cole, C. Coutures, M. F. Duran, J. Greenhill, 
F. Jablonski, U. Marboeuf, E. Martioli, E. Pedretti, O. Pejcha, P. Rojo, M. D. Albrow, S. Brillant, M. Bode, D. M. Bramich, M. J. Burgdorf, J. A. R. Caldwell, H. Calitz, E. Corrales, S. Dieters, D. Dominis Prester, J. Donatowicz, K. Hill, M. Hoffman, K. Horne, U. G. Jørgensen, N. Kains, S. Kane, J. B. Marquette, R. Martin, P. Meintjes, J. Menzies, K. R. Pollard, K. C. Sahu, C. Snodgrass, I. Steele, R. Street, Y. Tsapras, J. Wambsganss, A. Williams, M. Zub, PLANET Collaboration, M. K. Szymański, M. Kubiak, G. Pietrzyński, I. Soszyński, O. Szewczyk, L. Wyrzykowski, K. Ulaczyk, OGLE Collaboration, W. Allen, G. W. Christie, D. L. DePoy, B. S. Gaudi, C. Han, J. Janczak, C.-U. Lee, J. McCormick, F. Mallia, B. Monard, T. Natusch, B.-G. Park, R. W. Pogge, R. Santallo, and $\mu$ FUN Collaboration, A Cold Neptune-Mass Planet OGLE-2007-BLG-368Lb: Cold Neptunes Are Common, ApJ 710 (Feb., 2010) 1641-1653, arXiv:0912.1171].

[25] A. Gould, S. Dong, B. S. Gaudi, A. Udalski, I. A. Bond, J. Greenhill, R. A. Street, M. Dominik, T. Sumi, M. K. Szymański, C. Han, W. Allen, G. Bolt, M. Bos, G. W. Christie, D. L. DePoy, J. Drummond, J. D. Eastman, A. Gal-Yam, D. Higgins, J. Janczak, S. Kaspi, S. Kozłowski, C.-U. Lee, F. Mallia, A. Maury, D. Maoz, J. McCormick, L. A. G. Monard, D. Moorhouse, N. Morgan, T. Natusch, E. O. Ofek, B.-G. Park, R. W. Pogge, D. Polishook, R. Santallo, A. Shporer, O. Spector, G. Thornley, J. C. Yee, $\mu$ FUN Collaboration, M. Kubiak, G. Pietrzyński, I. Soszyński, O. Szewczyk, L. Wyrzykowski, K. Ulaczyk, R. Poleski, OGLE Collaboration, F. Abe, D. P. Bennett, C. S. Botzler, D. Douchin, M. Freeman, A. Fukui, K. Furusawa, J. B. Hearnshaw, S. Hosaka, Y. Itow, K. Kamiya, P. M. Kilmartin, A. Korpela, W. Lin, C. H. Ling, S. Makita, K. Masuda, Y. Matsubara, N. Miyake, Y. Muraki, M. Nagaya, K. Nishimoto, K. Ohnishi, T. Okumura, Y. C. Perrott, L. Philpott, N. Rattenbury, T. Saito, T. Sako, D. J. Sullivan, W. L. Sweatman, P. J. Tristram, E. von Seggern, P. C. M. Yock, MOA Collaboration, M. Albrow, V. Batista, J. P. Beaulieu, S. Brillant, J. Caldwell, J. J. Calitz, A. Cassan, A. Cole, K. Cook, C. Coutures, S. Dieters, D. Dominis Prester, J. Donatowicz, P. Fouqué, K. Hill, M. Hoffman, F. Jablonski, S. R. Kane, N. Kains, D. Kubas, J.-B. Marquette, R. Martin, E. Martioli, P. Meintjes, J. Menzies, E. Pedretti, K. Pollard, K. C. Sahu, C. Vinter, J. Wambsganss, R. Watson, A. Williams, M. Zub, PLANET Collaboration, A. Allan, M. F. Bode, D. M. Bramich, M. J. Burgdorf, N. Clay, S. Fraser, E. Hawkins, K. Horne, E. Kerins, T. A. Lister, C. Mottram, E. S. Saunders, C. Snodgrass, I. A. Steele, Y. Tsapras, RoboNet Collaboration, U. G. Jørgensen, T. Anguita, V. Bozza, S. Calchi Novati, K. Harpsøe, T. C. Hinse, M. Hundertmark, P. Kjærgaard, C. Liebig, L. Mancini, G. Masi, M. Mathiasen, S. Rahvar, D. Ricci, G. Scarpetta, J. Southworth, J. Surdej, C. C. Thöne, and MiNDSTEp Consortium, Frequency of Solar-like Systems and of Ice and Gas Giants Beyond the Snow Line from High-magnification Microlensing Events in 2005-2008, ApJ 720 (Sept., 2010) 1073-1089, arXiv: 1001.0572.

[26] A. Cassan, D. Kubas, J.-P. Beaulieu, M. Dominik, K. Horne, J. Greenhill, J. Wambsganss, J. Menzies, A. Williams, U. G. Jørgensen, A. Udalski, D. P. Bennett, M. D. Albrow, V. Batista, S. Brillant, J. A. R. Caldwell, A. Cole, C. Coutures, K. H. Cook, S. Dieters, D. D. Prester, J. Donatowicz, P. Fouqué, K. Hill, N. Kains, S. Kane, J.-B. Marquette, R. Martin, K. R. Pollard, K. C. Sahu, C. Vinter, D. Warren, B. Watson, M. Zub, T. Sumi, M. K. Szymański, M. Kubiak, R. Poleski, I. Soszynski, K. Ulaczyk, G. Pietrzyński, and L. Wyrzykowski, One or more bound planets per Milky Way star from microlensing observations, Nature 481 (Jan., 2012) 167-169, [arXiv:1202.0903].

[27] Y. Shvartzvald, D. Maoz, A. Udalski, T. Sumi, M. Friedmann, S. Kaspi, R. Poleski, M. K. Szymański, J. Skowron, S. Kozłowski, L. Wyrzykowski, P. Mróz, P. Pietrukowicz, G. Pietrzyński, I. Soszyński, K. Ulaczyk, F. Abe, R. K. Barry, D. P. Bennett, A. Bhattacharya, I. A. Bond, M. Freeman, K. Inayama, Y. Itow, N. Koshimoto, C. H. Ling, K. Masuda, A. Fukui, Y. Matsubara, Y. Muraki, K. Ohnishi, N. J. Rattenbury, T. Saito, D. J. Sullivan, D. Suzuki, P. J. Tristram, Y. Wakiyama, and A. Yonehara, The frequency of snowline-region planets from four-years of OGLE-MOA-Wise second-generation microlensing, ArXiv e-prints (Oct., 2015) [arXiv:1510.0429]. 
[28] A. Udalski, M. K. Szymański, and G. Szymański, OGLE-IV: Fourth Phase of the Optical Gravitational Lensing Experiment, Acta Astron. 65 (Mar., 2015) 1-38, arXiv: 1504.0596.

[29] T. Sako, T. Sekiguchi, M. Sasaki, K. Okajima, F. Abe, I. A. Bond, J. B. Hearnshaw, Y. Itow, K. Kamiya, P. M. Kilmartin, K. Masuda, Y. Matsubara, Y. Muraki, N. J. Rattenbury, D. J. Sullivan, T. Sumi, P. Tristram, T. Yanagisawa, and P. C. M. Yock, MOA-cam3: a wide-field mosaic CCD camera for a gravitational microlensing survey in New Zealand, Experimental Astronomy 22 (Oct., 2008) 51-66, arXiv:0804.0653.

[30] Y. Shvartzvald and D. Maoz, Second-generation microlensing planet surveys: a realistic simulation, MNRAS 419 (Feb., 2012) 3631-3640, [arXiv:1107.5809].

[31] B.-G. Park, S.-L. Kim, J. W. Lee, B.-C. Lee, C.-U. Lee, C. Han, M. Kim, D.-S. Moon, H.-K. Moon, S.-C. Rey, E.-C. Sung, and H. Sung, Korea Microlensing Telescope Network: science cases, in Society of Photo-Optical Instrumentation Engineers (SPIE) Conference Series, vol. 8444 of Society of Photo-Optical Instrumentation Engineers (SPIE) Conference Series, p. 47, Sept., 2012.

[32] C. B. Henderson, B. S. Gaudi, C. Han, J. Skowron, M. T. Penny, D. Nataf, and A. P. Gould, Optimal Survey Strategies and Predicted Planet Yields for the Korean Microlensing Telescope Network, ApJ 794 (Oct., 2014) 52, arXiv:1406.2316.

[33] C. Han, A. Udalski, J.-Y. Choi, J. C. Yee, A. Gould, G. Christie, T.-G. Tan, M. K. Szymański, M. Kubiak, I. Soszyński, G. Pietrzyński, R. Poleski, K. Ulaczyk, P. Pietrukowicz, S. Kozłowski, J. Skowron, L. Wyrzykowski, OGLE Collaboration, L. A. Almeida, V. Batista, D. L. Depoy, S. Dong, J. Drummond, B. S. Gaudi, K.-H. Hwang, F. Jablonski, Y.-K. Jung, C.-U. Lee, J.-R. Koo, J. McCormick, L. A. G. Monard, T. Natusch, H. Ngan, H. Park, R. W. Pogge, I. Porritt, I.-G. Shin, and $\mu$ FUN Collaboration, The Second Multiple-planet System Discovered by Microlensing: OGLE-2012-BLG-0026Lb, c-A Pair of Jovian Planets beyond the Snow Line, ApJL 762 (Jan., 2013) L28, arXiv:1210.4265].

[34] R. Poleski, A. Udalski, S. Dong, M. K. Szymański, I. Soszyński, M. Kubiak, G. Pietrzyński, S. Kozłowski, P. Pietrukowicz, K. Ulaczyk, J. Skowron, L. Wyrzykowski, and A. Gould, Super-massive Planets around Late-type Stars - the Case of OGLE-2012-BLG-0406Lb, ApJ 782 (Feb., 2014) 47, [arXiv:1307.4084].

[35] Y. Tsapras, J.-Y. Choi, R. A. Street, C. Han, V. Bozza, A. Gould, M. Dominik, J.-P. Beaulieu, A. Udalski, U. G. Jørgensen, T. Sumi, D. M. Bramich, P. Browne, K. Horne, M. Hundertmark, S. Ipatov, N. Kains, C. Snodgrass, I. A. Steele, RoboNet Collaboration, K. A. Alsubai, J. M. Andersen, S. Calchi Novati, Y. Damerdji, C. Diehl, A. Elyiv, E. Giannini, S. Hardis, K. Harpsøe, T. C. Hinse, D. Juncher, E. Kerins, H. Korhonen, C. Liebig, L. Mancini, M. Mathiasen, M. T. Penny, M. Rabus, S. Rahvar, G. Scarpetta, J. Skottfelt, J. Southworth, J. Surdej, J. Tregloan-Reed, C. Vilela, J. Wambsganss, MiNDSTEp Collaboration, J. Skowron, R. Poleski, S. Kozłowski, L. Wyrzykowski, M. K. Szymański, M. Kubiak, P. Pietrukowicz, G. Pietrzyński, I. Soszyński, K. Ulaczyk, OGLE Collaboration, M. D. Albrow, E. Bachelet, R. Barry, V. Batista, A. Bhattacharya, S. Brillant, J. A. R. Caldwell, A. Cassan, A. Cole, E. Corrales, C. Coutures, S. Dieters, D. Dominis Prester, J. Donatowicz, P. Fouqué, J. Greenhill, S. R. Kane, D. Kubas, J.-B. Marquette, J. Menzies, C. Père, K. R. Pollard, M. Zub, PLANET Collaboration, G. Christie, D. L. DePoy, S. Dong, J. Drummond, B. S. Gaudi, C. B. Henderson, K. H. Hwang, Y. K. Jung, A. Kavka, J.-R. Koo, C.-U. Lee, D. Maoz, L. A. G. Monard, T. Natusch, H. Ngan, H. Park, R. W. Pogge, I. Porritt, I.-G. Shin, Y. Shvartzvald, T. G. Tan, J. C. Yee, $\mu$ FUN Collaboration, F. Abe, D. P. Bennett, I. A. Bond, C. S. Botzler, M. Freeman, A. Fukui, D. Fukunaga, Y. Itow, N. Koshimoto, C. H. Ling, K. Masuda, Y. Matsubara, Y. Muraki, S. Namba, K. Ohnishi, N. J. Rattenbury, T. Saito, D. J. Sullivan, W. L. Sweatman, 
D. Suzuki, P. J. Tristram, N. Tsurumi, K. Wada, N. Yamai, P. C. M. Yock, A. Yonehara, and The MOA Collaboration, A Super-Jupiter Orbiting a Late-type Star: A Refined Analysis of Microlensing Event OGLE-2012-BLG-0406, ApJ 782 (Feb., 2014) 48, [arXiv:1310.2428].

[36] T. Sumi, K. Kamiya, D. P. Bennett, I. A. Bond, F. Abe, C. S. Botzler, A. Fukui, K. Furusawa, J. B. Hearnshaw, Y. Itow, P. M. Kilmartin, A. Korpela, W. Lin, C. H. Ling, K. Masuda, Y. Matsubara, N. Miyake, M. Motomura, Y. Muraki, M. Nagaya, S. Nakamura, K. Ohnishi, T. Okumura, Y. C. Perrott, N. Rattenbury, T. Saito, T. Sako, D. J. Sullivan, W. L. Sweatman, P. J. Tristram, A. Udalski, M. K. Szymański, M. Kubiak, G. Pietrzyński, R. Poleski, I. Soszyński, L. Wyrzykowski, K. Ulaczyk, and Microlensing Observations in Astrophysics (MOA) Collaboration, Unbound or distant planetary mass population detected by gravitational microlensing, Nature 473 (May, 2011) 349-352, [arXiv:1105.3544].

[37] J. Yoo, D. L. DePoy, A. Gal-Yam, B. S. Gaudi, A. Gould, C. Han, Y. Lipkin, D. Maoz, E. O. Ofek, B.-G. Park, R. W. Pogge, M. K. Szymański, A. Udalski, O. Szewczyk, M. Kubiak, K. Żebruń, G. Pietrzyński, I. Soszyński, and L. Wyrzykowski, Constraints on Planetary Companions in the Magnification A=256 Microlensing Event OGLE-2003-BLG-423, ApJ 616 (Dec., 2004) 1204-1214, [astro-ph/].

[38] M. Honma, MACHO Mass Determination Based on Space Telescope Observation, ApJL 517 (May, 1999) L35-L38, astro-ph/9903364.

[39] A. Gould, Geosynchronous Microlens Parallaxes, ApJL 763 (Feb., 2013) L35, arXiv:1211.6384].

[40] A. Gould and K. Horne, Kepler-like Multi-plexing for Mass Production of Microlens Parallaxes, ApJL 779 (Dec., 2013) L28, [arXiv:1310.4208].

[41] S. Refsdal, On the possibility of determining the distances and masses of stars from the gravitational lens effect, MNRAS 134 (1966) 315.

[42] A. Gould, Proper motions of MACHOs, ApJL 421 (Feb., 1994) L71-L74.

[43] A. Gould, A. Udalski, B. Monard, K. Horne, S. Dong, N. Miyake, K. Sahu, D. P. Bennett, L. Wyrzykowski, I. Soszyński, M. K. Szymański, M. Kubiak, G. Pietrzyński, O. Szewczyk, K. Ulaczyk, OGLE Collaboration, W. Allen, G. W. Christie, D. L. DePoy, B. S. Gaudi, C. Han, C.-U. Lee, J. McCormick, T. Natusch, B.-G. Park, R. W. Pogge, $\mu$ FUN Collaboration, A. Allan, M. F. Bode, D. M. Bramich, M. J. Burgdorf, M. Dominik, S. N. Fraser, E. Kerins, C. Mottram, C. Snodgrass, I. A. Steele, R. Street, Y. Tsapras, RoboNet Collaboration, F. Abe, I. A. Bond, C. S. Botzler, A. Fukui, K. Furusawa, J. B. Hearnshaw, Y. Itow, K. Kamiya, P. M. Kilmartin, A. Korpela, W. Lin, C. H. Ling, K. Masuda, Y. Matsubara, Y. Muraki, M. Nagaya, K. Ohnishi, T. Okumura, Y. C. Perrott, N. Rattenbury, T. Saito, T. Sako, L. Skuljan, D. J. Sullivan, T. Sumi, W. L. Sweatman, P. J. Tristram, P. C. M. Yock, MOA Collaboration, M. Albrow, J. P. Beaulieu, C. Coutures, H. Calitz, J. Caldwell, P. Fouque, R. Martin, A. Williams, and PLANET Collaboration, The Extreme Microlensing Event OGLE-2007-BLG-224: Terrestrial Parallax Observation of a Thick-Disk Brown Dwarf, ApJL 698 (June, 2009) L147-L151, arXiv:0904.0249.

[44] J. C. Yee, A. Udalski, T. Sumi, S. Dong, S. Kozłowski, J. C. Bird, A. Cole, D. Higgins, J. McCormick, L. A. G. Monard, D. Polishook, A. Shporer, O. Spector, M. K. Szymański, M. Kubiak, G. Pietrzyński, I. Soszyński, O. Szewczyk, K. Ulaczyk, L. Wyrzykowski, R. Poleski, OGLE Collaboration, W. Allen, M. Bos, G. W. Christie, D. L. DePoy, J. D. Eastman, B. S. Gaudi, A. Gould, C. Han, S. Kaspi, C.-U. Lee, F. Mallia, A. Maury, D. Maoz, T. Natusch, B.-G. Park, R. W. Pogge, R. Santallo, $\mu$ FUN Collaboration, F. Abe, I. A. Bond, A. Fukui, K. Furusawa, J. B. Hearnshaw, S. Hosaka, Y. Itow, 
K. Kamiya, A. V. Korpela, P. M. Kilmartin, W. Lin, C. H. Ling, S. Makita, K. Masuda, Y. Matsubara, N. Miyake, Y. Muraki, M. Nagaya, K. Nishimoto, K. Ohnishi, Y. C. Perrott, N. J. Rattenbury, T. Sako, T. Saito, L. Skuljan, D. J. Sullivan, W. L. Sweatman, P. J. Tristram, P. C. M. Yock, MOA Collaboration, M. D. Albrow, V. Batista, P. Fouqué, J.-P. Beaulieu, D. P. Bennett, A. Cassan, J. Comparat, C. Coutures, S. Dieters, J. Greenhill, K. Horne, N. Kains, D. Kubas, R. Martin, J. Menzies, J. Wambsganss, A. Williams, M. Zub, and PLANET Collaboration, Extreme Magnification Microlensing Event OGLE-2008-BLG-279: Strong Limits on Planetary Companions to the Lens Star, ApJ 703 (Oct., 2009) 2082-2090, arXiv:0907.5411].

[45] J.-Y. Choi, C. Han, A. Udalski, T. Sumi, B. S. Gaudi, A. Gould, D. P. Bennett, M. Dominik, J.-P. Beaulieu, Y. Tsapras, V. Bozza, F. Abe, I. A. Bond, C. S. Botzler, P. Chote, M. Freeman, A. Fukui, K. Furusawa, Y. Itow, C. H. Ling, K. Masuda, Y. Matsubara, N. Miyake, Y. Muraki, K. Ohnishi, N. J. Rattenbury, T. Saito, D. J. Sullivan, K. Suzuki, W. L. Sweatman, D. Suzuki, S. Takino, P. J. Tristram, K. Wada, P. C. M. Yock, MOA Collaboration, M. K. Szymański, M. Kubiak, G. Pietrzyński, I. Soszyński, J. Skowron, S. Kozłowski, R. Poleski, K. Ulaczyk, L. Wyrzykowski, P. Pietrukowicz, OGLE Collaboration, L. A. Almeida, D. L. DePoy, S. Dong, E. Gorbikov, F. Jablonski, C. B. Henderson, K.-H. Hwang, J. Janczak, Y.-K. Jung, S. Kaspi, C.-U. Lee, U. Malamud, D. Maoz, D. McGregor, J. A. Muñoz, B.-G. Park, H. Park, R. W. Pogge, Y. Shvartzvald, I.-G. Shin, J. C. Yee, $\mu$ FUN Collaboration, K. A. Alsubai, P. Browne, M. J. Burgdorf, S. Calchi Novati, P. Dodds, X.-S. Fang, F. Finet, M. Glitrup, F. Grundahl, S.-H. Gu, S. Hardis, K. Harpsøe, T. C. Hinse, A. Hornstrup, M. Hundertmark, J. Jessen-Hansen, U. G. Jrgensen, N. Kains, E. Kerins, C. Liebig, M. N. Lund, M. Lundkvist, G. Maier, L. Mancini, M. Mathiasen, M. T. Penny, S. Rahvar, D. Ricci, G. Scarpetta, J. Skottfelt, C. Snodgrass, J. Southworth, J. Surdej, J. Tregloan-Reed, J. Wambsganss, O. Wertz, F. Zimmer, T. MiNDSTEp Consortium, M. D. Albrow, E. Bachelet, V. Batista, S. Brillant, A. Cassan, A. A. Cole, C. Coutures, S. Dieters, D. Dominis Prester, J. Donatowicz, P. Fouqué, J. Greenhill, D. Kubas, J.-B. Marquette, J. W. Menzies, K. C. Sahu, M. Zub, PLANET Collaboration, D. M. Bramich, K. Horne, I. A. Steele, R. A. Street, and RoboNet Collaboration, Microlensing Discovery of a Population of Very Tight, Very Low Mass Binary Brown Dwarfs, ApJ 768 (May, 2013) 129, [arXiv:1302.4169].

[46] Y. K. Jung, A. Udalski, T. Sumi, C. Han, A. Gould, J. Skowron, S. Kozłowski, R. Poleski, L. Wyrzykowski, M. K. Szymański, G. Pietrzyński, I. Soszyński, K. Ulaczyk, P. Pietrukowicz, P. Mróz, M. Kubiak, OGLE Collaboration, F. Abe, D. P. Bennett, I. A. Bond, C. S. Botzler, M. Freeman, A. Fukui, D. Fukunaga, Y. Itow, N. Koshimoto, P. Larsen, C. H. Ling, K. Masuda, Y. Matsubara, Y. Muraki, S. Namba, K. Ohnishi, L. Philpott, N. J. Rattenbury, T. Saito, D. J. Sullivan, D. Suzuki, P. J. Tristram, N. Tsurumi, K. Wada, N. Yamai, P. C. M. Yock, A. Yonehara, The MOA Collaboration, M. Albrow, J.-Y. Choi, D. L. DePoy, B. S. Gaudi, K.-H. Hwang, C.-U. Lee, H. Park, S. Owen, R. W. Pogge, I.-G. Shin, J. C. Yee, and The $\mu$ FUN Collaboration, OGLE-2013-BLG-0102LA,B: Microlensing Binary with Components at Star/Brown Dwarf and Brown Dwarf/Planet Boundaries, ApJ 798 (Jan., 2015) 123, arXiv:1407.7926.

[47] S. Calchi Novati, A. Gould, A. Udalski, J. W. Menzies, I. A. Bond, Y. Shvartzvald, R. A. Street, M. Hundertmark, C. A. Beichman, J. C. Yee, S. Carey, R. Poleski, J. Skowron, S. Kozłowski, P. Mróz, P. Pietrukowicz, G. Pietrzyński, M. K. Szymański, I. Soszyński, K. Ulaczyk, L. Wyrzykowski, The OGLE collaboration, M. Albrow, J. P. Beaulieu, J. A. R. Caldwell, A. Cassan, C. Coutures, C. Danielski, D. Dominis Prester, J. Donatowicz, K. Lončarić, A. McDougall, J. C. Morales, C. Ranc, W. Zhu, The PLANET collaboration, F. Abe, R. K. Barry, D. P. Bennett, A. Bhattacharya, D. Fukunaga, K. Inayama, N. Koshimoto, S. Namba, T. Sumi, D. Suzuki, P. J. Tristram, Y. Wakiyama, A. Yonehara, The MOA collaboration, D. Maoz, S. Kaspi, M. Friedmann, Wise group, E. Bachelet, R. Figuera Jaimes, D. M. Bramich, Y. Tsapras, K. Horne, C. Snodgrass, J. Wambsganss, I. A. Steele, 
N. Kains, RoboNet collaboration, V. Bozza, M. Dominik, U. G. Jørgensen, K. A. Alsubai, S. Ciceri, G. D’Ago, T. Haugbølle, F. V. Hessman, T. C. Hinse, D. Juncher, H. Korhonen, L. Mancini, A. Popovas, M. Rabus, S. Rahvar, G. Scarpetta, R. W. Schmidt, J. Skottfelt, J. Southworth, D. Starkey, J. Surdej, O. Wertz, M. Zarucki, MiNDSTEp consortium, B. S. Gaudi, R. W. Pogge, D. L. DePoy, and $\mu$ FUN collaboration, Pathway to the Galactic Distribution of Planets: Combined Spitzer and Ground-Based Microlens Parallax Measurements of 21 Single-Lens Events, ApJ 804 (May, 2015) 20, [arXiv:1411.7378].

[48] S. Calchi Novati, A. Gould, J. C. Yee, C. Beichman, G. Bryden, S. Carey, M. Fausnaugh, B. S. Gaudi, C. B. Henderson, R. W. Pogge, Y. Shvartzvald, B. Wibking, W. Zhu, Spitzer team, A. Udalski, R. Poleski, M. Pawlak, M. K. Szymański, J. Skowron, P. Mróz, S. Kozłowski, L. Wyrzykowski, P. Pietrukowicz, G. Pietrzyński, I. Soszyński, K. Ulaczyk, and OGLE group, Spitzer IRAC Photometry for Time Series in Crowded Fields, ApJ 814 (Dec., 2015) 92, [arXiv:1509.0003].

[49] J. C. Yee, A. Udalski, S. Calchi Novati, A. Gould, S. Carey, R. Poleski, B. S. Gaudi, R. W. Pogge, J. Skowron, S. Kozłowski, P. Mróz, P. Pietrukowicz, G. Pietrzyński, M. K. Szymański, I. Soszyński, K. Ulaczyk, and L. Wyrzykowski, First Space-based Microlens Parallax Measurement of an Isolated Star: Spitzer Observations of OGLE-2014-BLG-0939, ApJ 802 (Apr., 2015) 76, [arXiv:1410.5429].

[50] R. A. Street, A. Udalski, S. Calchi Novati, M. P. G. Hundertmark, W. Zhu, A. Gould, J. Yee, Y. Tsapras, D. P. Bennett, T. RoboNet Project, M. Consortium, U. G. Jorgensen, M. Dominik, M. I. Andersen, E. Bachelet, V. Bozza, D. M. Bramich, M. J. Burgdorf, A. Cassan, S. Ciceri, G. D’Ago, S. Dong, D. F. Evans, S.-h. Gu, H. Harkonnen, T. C. Hinse, K. Horne, R. Figuera Jaimes, N. Kains, E. Kerins, H. Korhonen, M. Kuffmeier, L. Mancini, J. Menzies, S. Mao, N. Peixinho, A. Popovas, M. Rabus, S. Rahvar, C. Ranc, R. Tronsgaard Rasmussen, G. Scarpetta, R. Schmidt, J. Skottfelt, C. Snodgrass, J. Southworth, I. A. Steele, J. Surdej, E. Unda-Sanzana, P. Verma, C. von Essen, J. Wambsganss, Y.-B. Wang, O. Wertz, T. OGLE Project, R. Poleski, M. Pawlak, M. K. Szymanski, J. Skowron, P. Mroz, S. Kozlowski, L. Wyrzykowski, P. Pietrukowicz, G. Pietrzynski, I. Soszynski, K. Ulaczyk, The Spitzer Team C. Beichman, G. Bryden, S. Carey, B. S. Gaudi, C. Henderson, R. W. Pogge, Y. Shvartzvald, The MOA Collaboration, F. Abe, Y. Asakura, A. Bhattacharya, I. A. Bond, M. Donachie, M. Freeman, A. Fukui, Y. Hirao, K. Inayama, Y. Itow, N. Koshimoto, M. C. A. Li, C. H. Ling, K. Masuda, Y. Matsubara, Y. Muraki, M. Nagakane, T. Nishioka, K. Ohnishi, H. Oyokawa, N. Rattenbury, T. Saito, A. Sharan, D. J. Sullivan, T. Sumi, D. Suzuki, P., J. Tristram, Y. Wakiyama, A. Yonehara, KMTNet Modeling Team C. Han, J.-Y. Choi, H. Park, Y. K. Jung, and I.-G. Shin, Spitzer Parallax of OGLE-2015-BLG-0966: A Cold Neptune in the Galactic Disk, ArXiv e-prints (Aug., 2015) [arXiv:1508.0702].

[51] Y. Shvartzvald, A. Udalski, A. Gould, C. Han, V. Bozza, M. Friedmann, M. Hundertmark, C. Beichman, G. Bryden, S. Calchi Novati, S. Carey, M. Fausnaugh, B. S. Gaudi, C. B. Henderson, T. Kerr, R. W. Pogge, W. Varricatt, B. Wibking, J. C. Yee, W. Zhu, R. Poleski, M. Pawlak, M. K. Szymański, J. Skowron, P. Mróz, S. Kozłowski, L. Wyrzykowski, P. Pietrukowicz, G. Pietrzyński, I. Soszyński, K. Ulaczyk, J.-Y. Choi, H. Park, Y. K. Jung, I.-G. Shin, M. D. Albrow, B.-G. Park, S.-L. Kim, C.-U. Lee, S.-M. Cha, D.-J. Kim, Y. Lee, D. Maoz, S. Kaspi, R. A. Street, Y. Tsapras, E. Bachelet, M. Dominik, D. M. Bramich, K. Horne, C. Snodgrass, I. A. Steele, J. Menzies, R. Figuera Jaimes, J. Wambsganss, R. Schmidt, A. Cassan, C. Ranc, S. Mao, S. Dong, G. D’Ago, G. Scarpetta, P. Verma, U. G. Jørgensen, E. Kerins, and J. Skottfelt, Spitzer Microlens Measurement of a Massive Remnant in a Well-Separated Binary, ArXiv e-prints (Aug., 2015) arXiv:1508.0663.

[52] J. C. Yee, A. Gould, C. Beichman, S. Calchi Novati, S. Carey, B. S. Gaudi, C. B. Henderson, D. Nataf, M. Penny, Y. Shvartzvald, and W. Zhu, Criteria for Sample Selection to Maximize Planet 
Sensitivity and Yield from Space-Based Microlens Parallax Surveys, ApJ 810 (Sept., 2015) 155, [arXiv:1505.0001].

[53] A. Gould, J. Yee, and S. Carey, "Galactic Distribution of Planets From High-Magnification Microlensing Events." Spitzer Proposal, Oct., 2015.

[54] A. Gould, J. Yee, and S. Carey, "Degeneracy Breaking for K2 Microlens Parallaxes." Spitzer Proposal, Oct., 2015.

[55] e. Henderson, C. in prep (2016).

[56] D. Spergel, N. Gehrels, C. Baltay, D. Bennett, J. Breckinridge, M. Donahue, A. Dressler, B. S. Gaudi, T. Greene, O. Guyon, C. Hirata, J. Kalirai, N. J. Kasdin, B. Macintosh, W. Moos, S. Perlmutter, M. Postman, B. Rauscher, J. Rhodes, Y. Wang, D. Weinberg, D. Benford, M. Hudson, W.-S. Jeong, Y. Mellier, W. Traub, T. Yamada, P. Capak, J. Colbert, D. Masters, M. Penny, D. Savransky, D. Stern, N. Zimmerman, R. Barry, L. Bartusek, K. Carpenter, E. Cheng, D. Content, F. Dekens, R. Demers, K. Grady, C. Jackson, G. Kuan, J. Kruk, M. Melton, B. Nemati, B. Parvin, I. Poberezhskiy, C. Peddie, J. Ruffa, J. K. Wallace, A. Whipple, E. Wollack, and F. Zhao, Wide-Field InfrarRed Survey Telescope-Astrophysics Focused Telescope Assets WFIRST-AFTA 2015 Report, ArXiv e-prints (Mar., 2015) arXiv:1503.0375.

[57] J. C. Yee, Lens Masses and Distances from Microlens Parallax and Flux, ApJL 814 (Nov., 2015) L11, [arXiv:1509.0504]. 